\title{
Contraception concerns, utilization and counseling needs of women with a history of breast cancer: a qualitative study
}

This article was published in the following Dove Press journal:

International Journal of Women's Health

21 July 2017

Number of times this article has been viewed

\author{
Sheila K Mody' \\ Danielle M Panelli' \\ Avanthi Hulugalle' \\ $\mathrm{H}$ Irene $\mathrm{Su}^{2}$ \\ Jessica R Gorman ${ }^{3}$ \\ 'Division of Family Planning, \\ Department of Reproductive \\ Medicine, University of California, San \\ Diego, San Diego, CA, USA; 'Division \\ of Reproductive Endocrinology \\ and Infertility, Department of \\ Reproductive Medicine, University \\ of California, San Diego, San Diego, \\ CA, USA; ${ }^{3}$ College of Public Health \\ and Human Sciences, Oregon State \\ University, Corvallis, OR, USA
}

Introduction: Reproductive-aged women who have had breast cancer face unique challenges when it comes to making decisions about contraception. Clinical guidelines indicate that patients should avoid pregnancy during cancer treatment, however the extent of contraception counseling and utilization after diagnosis has not been well studied.

Methods: We conducted three focus groups and one semi-structured interview with women between the ages of 18 and 50 years old diagnosed with breast cancer within the last 5 years. We used clinic- and population-based recruitment methods to identify participants. Participants were asked a series of open-ended questions regarding their contraception decision-making and concerns at the time of their diagnosis, during treatment and afterward. We analyzed data to identify themes among participant responses.

Results: A total of 10 women participated. We identified the following themes: 1) doctors treating cancer do not focus on reproductive health issues; 2) there is misinformation and lack of information on contraceptive options and risks; 3) women fear unintended pregnancy but have limited guidance on prevention; 4) peers are a trusted source of contraception information; and 5) information about contraception should be provided soon after diagnosis.

Conclusion: Reproductive-aged breast cancer survivors have unique contraceptive concerns and may not be adequately informed about their contraceptive options. The results of this study may help guide and improve contraceptive counseling services for breast cancer survivors.

Keywords: breast cancer survivors, contraception utilization, contraception counseling, peer counseling

\section{Introduction}

Women diagnosed with breast cancer who are undergoing treatment or adjuvant therapy are advised to avoid pregnancy due to the potential detrimental impact on their health as well as potential impact on the fetus. ${ }^{1}$ However, most are also told to avoid estrogen and progestogen/progestin hormones, which are components of many modern contraceptive methods. In fact, the Centers for Disease Control and Prevention discourages use of combined contraceptives in patients with any active cancer, not just breast cancer, within 6 months of diagnosis or treatment due to the increase in venous thromboembolism risk. Although clinicians may assume that these patients are practicing abstinence, many breast cancer patients remain sexually active during and after chemotherapy, and many use condoms rather than highly effective contraception methods. ${ }^{2,3}$ This introduces a higher risk of unintended pregnancy, as the rate of unintended pregnancy with typical use of condoms has been estimated to be $18 \%$ in the general population. ${ }^{4}$ In contrast, the failure rate of the copper intrauterine device (IUD)
Division of Family Planning, Department of Reproductive Medicine, University of California, San Diego, 9300 Campus Point Drive, MC 7433, La Jolla, San Diego,

CA 92037, USA

Tel + I 858249 I205

Fax +I 8586577212

Email smody@ucsd.edu
International Journal of Women's Health 2017:9 507-5/2 Dovepress if in 8 http://dx,doi.org/10.2147/1JWH.S136120 (c) (1) (5) 2017 Mody et al. This work is published and licensed by Dove Medical Press limited. The full terms of this license are available at https://www.dovepress.com/terms.php cc) ${ }_{\mathrm{BY}} \mathrm{NC}$ and incorporate the Creative Commons Attribution - Non Commercial (unported, v3.0) License (http://creativecommons.org/licenses/by-nc/3.0/). By accessing the work you hereby accept the Terms. Non-commercial uses of the work are permitted without any further permission from Dove Medical Press Limited, provided the work is properly attributed. For permission for commercial use of this work, please see paragraphs 4.2 and 5 of our Terms (https://www.dovepress.com/terms.php). 
a non-hormonal method, is estimated to be $<1 \% .{ }^{4}$ According to the World Health Organization Medical Eligibility Criteria for Contraceptive Use, the recommended contraceptive methods for women with a history of breast cancer within 5 years are condoms or the copper IUD. ${ }^{5}$ Female cancer survivors are less likely to use highly effective methods of contraception compared to the general US population. ${ }^{4}$ However, little is know about the reasons behind both lower rates of using highly effective methods and choice of contraceptive methods.

Given the importance of using highly effective contraception to prevent unintended pregnancy especially during cancer treatment, we conducted an exploratory study to learn more about reproductive-aged breast cancer survivors' knowledge of contraceptive options, experiences obtaining contraceptive counseling, concerns about contraceptive use, and barriers to adopting highly effective contraception methods such as a copper IUD. Gaining a deeper understanding of survivors' experiences and concerns about contraception will benefit health care providers and researchers in identifying better approaches to help their patients make informed contraceptive decisions.

\section{Methods}

\section{Study design}

We utilized a qualitative research approach, including an option to participate in a focus group or individual interview, to collect in-depth information on breast cancer survivors' knowledge, experiences, and concerns about contraception. The study was approved by the Human Research Protection Program at the University of California, San Diego. All of the participants provided written informed consent.

\section{Sampling and eligibility criteria}

We recruited English-speaking, reproductive-aged female breast cancer survivors (aged 18-50 years) who were diagnosed with Stage 0-IV breast cancer within five years prior to study enrollment. Participants were recruited between 2013 and 2014 from the Moores Cancer Center at the University of California, San Diego and via the local Young Survival Coalition.

\section{Interviewing and data collection}

Focus groups included 2-5 participants each. Groups were purposefully small because of the sensitive subject matter. One participant elected to complete an individual semi-structured interview. The length of each focus group/ interview was approximately $60 \mathrm{~min}$.
We used a semi-structured guide with open-ended questions that were designed to encourage an in-depth discussion of participants' experiences both at the time of their cancer diagnosis and afterward. The guide included questions regarding: knowledge of contraception (eg, "What do you know about contraception options for breast cancer survivors?"); contraceptive counseling experiences (eg, "How would you summarize your experience with contraceptive counseling as a breast cancer survivor?'); feelings about contraception (eg, "How do you think most other young breast cancer survivors feel about contraception?'); and concerns about using a recommended method of contraception for breast cancer survivors (eg, "How do you feel about using a copper IUD for contraception?"). Both the focus groups and the structured interview were audio-recorded with participant permission. To ensure accuracy and avoid erroneous recall, we reviewed the recordings and compared them against the notes taken.

\section{Analysis}

The focus groups and interview were transcribed and imported into NVivo qualitative data analysis software (NVivo 10; QSR International, Melbourne, VIC, Australia). ${ }^{6}$ We used cross-case analysis to identify themes, combining answers from all participants and using these grouped answers to analyze responses. ${ }^{7}$ We used inductive analysis, allowing patterns and themes to emerge from the raw data. After each focus group or interview, the research team met to discuss the session and responses, evaluate the quality of the session, and to make improvements for future sessions. We used the following iterative strategy to analyze the data: 1) reading and re-reading qualitative data to begin developing codes; 2) coding data to begin developing themes and sub-themes; 3 ) identifying and noting categories and variations in themes; 4) reducing data to essential points; and 5) developing an overall final interpretation. ${ }^{8}$

The principal investigator and a coinvestigator collected the data. The coding and identification of themes was conducted separately by two coinvestigators. The two coinvestigators and the principal investigator came to a consensus about the final themes and sub-themes and confirmed that the themes accurately represented the discussions.

\section{Results}

\section{Study participants}

This study included 10 reproductive-aged sexually active women diagnosed with breast cancer within the prior 5 years. The age range of participants was 30-47 years (mean 40 years). Participants reported variable cancer stages: one 
ductal carcinoma in situ; three Stage 1 invasive ductal carcinoma; three Stage 2 invasive ductal carcinoma; and one metastatic. Two participants did not provide their disease stage. One participant reporting having a $B R C A$ mutation. The participants were racially and ethnically diverse, and most had at least a college degree. Participant characteristics are in Table 1.

\section{Themes}

The following five themes emerged from the data: 1) there is misinformation and lack of information on contraceptive options and risks; 2) women fear unintended pregnancy but have limited guidance on prevention; 3) doctors treating cancer do not focus on reproductive health issues; 4) peers are a trusted source of contraception information; and 5) information about contraception should be provided soon after diagnosis.

\section{Theme I: There is lack of information or} misinformation on contraceptive options and risks Many participants mentioned the lack of information that they were given regarding their contraceptive options. In addition, sometimes information seemed incorrect or incomplete.

I don't remember ever discussing contraception with my doctors.

I felt bad for other people who just got diagnosed. I would meet them at the breast cancer support group, and they would not have any idea of what they want or what they wanted to do or what their [contraceptive] options were. I was just like there's no, I mean there's like no information.

Table I Demographics

\begin{tabular}{ll}
\hline Participant characteristics & $\mathbf{N}(\%) \mathbf{( N = 1 0 )}$ \\
\hline Current age (years) & $2(20)$ \\
$30-35$ & $2(20)$ \\
$36-40$ & $5(50)$ \\
$41-45$ & $\mathrm{I}(10)$ \\
$46-50$ & \\
Race & $4(40)$ \\
White & $2(20)$ \\
Black & $2(20)$ \\
Hispanic & $2(20)$ \\
Asian/Pacific Islander & \\
Education & $\mathrm{I}(10)$ \\
High school graduate & $4(40)$ \\
College graduate & $3(30)$ \\
Master's degree & $2(20)$ \\
Other &
\end{tabular}

Some participants also received messages focused on avoiding pregnancy, but not specific contraceptive recommendations.

My doctor talked about it ... [I] needed to be sure not to get pregnant because of the chemo. So the first few appointments she was like, you're using contraception right, you're sure you're using contraception. So nothing beyond that ... she didn't tell me any choices, but she wanted me to be safe and use something so that I didn't get pregnant.

And similarly my oncologist sort of kind of scared that into me and was even doing pregnancy tests when I would come in for ... checkups.

Sub-theme IA: Women were specifically concerned about the safety of using a copper IUDs with magnetic resonance imaging (MRI)

Participants were confused about whether or not a copper IUD would be incompatible with their MRI scans. Some were advised against use of the copper IUD or were instructed to remove the copper IUD prior to an MRI scan.

I actually thought I was going to get an IUD a couple of weeks ago, but then some new research came out that because there's copper in one ... that could interfere with future MRIs.

I wish that I could do an IUD and not have to do a diaphragm. I would like to have the ease of something that you don't have to think about, whether or not that's a pill that you get used to taking every day or you know something that's inserted into your arm or your ... so like it's a little disappointing to me that I ... that my only option is either a condom or a diaphragm.

Sub-theme IB: Contraceptive discussions were often deferred to an obstetrician gynecologist (Ob/Gyn), but participants would prefer to receive that information from a member of their cancer care team

Overall, participants reported that when they asked their cancer care providers about contraception, they were referred to their $\mathrm{Ob} / \mathrm{Gyn}$ for specific recommendations.

My oncologist said you are not allowed to get pregnant. And then, and he might have mentioned several things that you could do but I do not really recall what ... and so he was just like I would just defer to the $\mathrm{Ob} / \mathrm{Gyn}$.

My oncologist just said you need to stop birth control pills and so then I went to my $\mathrm{Ob} / \mathrm{Gyn}$ to talk about other options. 
Participants discussed seeing an "amazing" number of doctors during their cancer treatment. Overall, women expressed a preference for one of these providers to offer contraceptive counseling, rather than going to yet another appointment. While there was a clear preference for contraceptive counseling to be integrated into their overall cancer care, there was no consensus on who should conduct the counseling.

\section{Theme 2: Women fear unintended pregnancy but have limited guidance on family planning options}

Women recalled being told not to get pregnant, but reported receiving little information on contraceptive options, resulting in a general fear about unintended pregnancy and risk to the baby.

There definitely needs to be a piece in there [what doctors say] for any type of cancer, I mean not just breast cancer about now that you have the big $\mathrm{C}$, now that you're looking at some possible treatments that may affect infertility, may affect your fertility and be dangerous to a fetus if you get pregnant, we need to discuss all of that, all of the family planning issues.

\section{Subtheme 2A: Awareness of the teratogenicity of chemotherapy and radiation}

Many of the participants were aware of the teratogenicity of some of the chemotherapeutics and hormone treatments. They were afraid of getting pregnant because these medications could be harmful to the baby.

She was just making sure that I understood that it was very serious that, you know you cannot, you know if you get pregnant you have to abort it because you're going to be doing chemo. I don't think she said the word like you have to abort it, but obviously I think it was implied that like okay so if you get pregnant then you have all these chemicals and that's not going to be good for the baby.

I heard that on tamoxifen you shouldn't be getting pregnant, so it was like should I do another form of barrier than just condoms, but my oncologist said that it was fine if I just did the condoms.

While most participants were informed of the teratogenic risk from their doctors, others had to connect the dots on their own.

I don't know if it's because I did not have chemo, but nobody ever told me that you need to make sure you don't get pregnant. I mean common sense kicked in and I knew that, but I went through radiation and nobody ever addressed that though really and I don't feel short changed because of it, but it might be good for some people to be told that.

\section{Theme 3: Doctors treating cancer do not focus on reproductive health issues}

Participants reported that there was lack of guidance on reproductive issues, including contraception and fertility issues. They reported that their cancer care providers focused primarily on treating cancer rather than addressing their reproductive health needs. Some women reported feeling abandoned on these issues. As one participant said, "I think oncologists deal with death every day. They only want to save your life. They don't really look outside of what quality of life you want to have for yourself or what you want to do."

\section{Theme 4: Peers are a trusted source of contraception information}

Women reported that they trusted the contraception information from fellow breast cancer survivors because it was based on personal experience, and they valued peer mentorship on reproductive health concerns.

So for me coming from a survivor, any information coming from a survivor gets like up here because they have actually gone through it so. Because a lot of the doctors that I had actually had never had cancer. You know they are very knowledgeable but they never like had it, never had it young. I mean and in terms of like I did not, I would rather it had come from a woman to tell you the truth ... it is just probably a personal preference but if it came from a woman and a survivor I will listen.

I love my oncologist and I feel I can speak ... openly about anything, but I think maybe, you know sitting down ... with other survivors who have been through the same process and around the same age I think ... It's a lot and nobody will understand unless they're in your same shoes.

Participants voiced a preference for peer counselors who also had medical knowledge, such as a nurse who was a breast cancer survivor.

[I would want to talk to] Somebody who is within the medical field, a nurse or another doctor, who's part of our team here and she's also a survivor ... somebody who's actually knowledgeable.

If we're talking about contraception and fertility, you know we all have really key things that are impacting us during that time and everyone's experience is so different ... so 
you know I would almost like a nurse or someone who is really knowledgeable.

\section{Theme 5: Information about contraception should be provided soon after diagnosis}

Several participants preferred early discussion of reproductive consequences of treatment and contraception counseling, in one of the first few visits with their oncologist. In many cases, women reported that the discussion did not happen early enough, leaving the women with anxiety when the topic came up at a later time.

I definitely think the oncologist should bring it up just right when they get your diagnosis and you start talking to people about what your [contraception] options are going to be that certainly should be in the conversation, especially if the doctor sees the patient and their age and asks ... do you have kids.

\section{Discussion}

We identified several main themes regarding the contraceptive counseling experiences, concerns and needs of the breast cancer survivors we interviewed. The most significant finding was that participants in this study indicated they wanted more contraceptive information sooner after their cancer diagnosis. Several of the participants voiced that they knew that they should not get pregnant after their diagnosis, but they did not know the safe and effective contraceptive methods they could utilize. They were fearful of hormonal contraceptive methods and believed that barrier methods, such as condoms, were their only option.

Participants were also uncertain about the safety of using a copper IUD if they required MRIs. This is an area of disconnect between some obstetrics and gynecology and radiology disciplines, especially when a 3 Telsa MRI machine is utilized instead of a 1.5 Telsa MRI machine. This is an area with limited published research. Berger-Kulemann et $\mathrm{al}^{9}$ demonstrated the safety of the copper intrauterine device with an ex vivo study (outside the body). No in vivo (within the body) research studies have been done. A case series study involving 18 women, evaluating patients with a Paragard IUD and a 3 Telsa MRI, suggests safety, with only one patient having a dislocation and another with a partial perforation (though the timing of both is unclear, as these may have been present prior to the MRI being done). ${ }^{9}$ Although the existing research suggests the safety of current MRIs with a copper IUD, oncologists may remain concerned and counsel their patients not to use the copper IUD. Clearly, there is a need for further research on this topic. Better communication among family planning specialists, radiologists and oncologists may ensure that patients receive consistent, accurate evidence-based counseling.

Despite the focus of this study being on contraceptive needs of this patient population, the desire for comprehensive reproductive health information, including on fertility, came up several times. Participants indicated the need for more fertility preservation and contraception information by knowledgeable clinicians soon after the diagnosis of breast cancer. There was strong interest in a peer counselor program to help meet these reproductive health informational needs. Similar peer counselor programs exist for HIV prenatal pregnant patients. ${ }^{10}$ In the study conducted by Richter et al, ${ }^{10}$ the peer intervention consisted of four antenatal and four postnatal small group sessions led by peer mentors. The peer mentoring focused on improving maternal and infant well-being. Ideally, participants in our study desired a breast cancer survivor peer counselor who was also a clinician with reproductive expertise. Patient navigators have been successfully utilized among breast cancer patients however patient navigators or peer mentors have not been used to specifically address reproductive health concerns. ${ }^{11}$ Based on the feedback from the participants in this study, adding reproductive health to an existing patient navigator program may be of benefit.

This study contributes to the emerging research on the family planning and contraceptive needs of reproductiveaged cancer survivors. There are very few existing studies on this topic. Güth et $\mathrm{al}^{12}$ conducted a survey of 100 breast cancer patients $<40$ years old and 101 medical oncologists regarding contraceptive counseling. This study found that most participants at the time of diagnosis were not using highly effective contraception. Only $8 \%$ of participants were using IUDs. Although $90 \%$ of oncologists reported using contraception prior to starting therapy, only $20 \%$ reported that they inform the patients that reliable contraception is needed prior to starting therapy, ask about which method is used during treatment and refer patients to a gynecologist for contraception counseling. ${ }^{12}$ Compared to the study done by Güth et al, our study gives more in-depth qualitative information about the contraceptive practices among breast cancer patients, however does not tell us in-depth qualitative information about the oncologist counseling.

There are some limitations of our study. Most notable was that the study size was small. In addition, while the participants were ethnically diverse, the majority had a college education and seemed well informed about their medical conditions. Inclusion of a more diverse education 
level participant population could have yielded different results. As with all qualitative studies, the results apply only to our participant population and therefore may not be generalizable.

The results of this study demonstrate the information gaps and areas for improvement in family planning education for reproductively aged breast cancer survivors. The findings provide preliminary data characterizing the level of contraception concerns across a diverse group of breast cancer survivors that will aid the development of effective interventions in the future. The major strength of this study was utilizing a qualitative research design which provides in-depth insight into the current contraceptive counseling breast cancer survivors receive and how these women envision improved contraceptive counseling.

\section{Acknowledgments}

We would like to thank our local Young Survivors Coalition and the Moore's Cancer Center for helping with the recruitment of participants.

The abstract of this paper was presented at the 2014 Oncofertility Conference in Chicago, IL, USA as a poster presentation with interim findings.

\section{Disclosure}

Dr Mody was partially supported by the UCSD Women's Reproductive Health Research NIH grant K12 HD001259. Dr Mody is also a Nexplanon trainer for Merck. In addition, Dr Mody is a consultant for Daré Bioscience, Inc. Pharmacist Dr Gorman was partially supported by American Cancer
Society grant 120500-PFT-11-008-01-CPPB. The authors report no other conflicts of interest in this work.

\section{References}

1. Braems G, Denys H, De Wever O, Cocquyt V, Van de Broecke R. Use of tamoxifen before and during pregnancy. Oncologist. 2011;16(11): $1547-1551$.

2. Cook-Andersen H, Komrokian S, DeMichele A, Su HI. Breast Cancer patients have lower rates of contraception use. Fertil Steril. 2011; 96(3):S201-S202.

3. Dominick SA, McLean MR, Whitcomb BW, et al. Contraceptive Practices Among Female Cancer Survivors of Reproductive Age. Obstet Gynecol. 2015;126(3):498-507.

4. Trussell J. Contraceptive Efficacy. In: Hatcher RA, Trussell J, Nelson AL, Cates W, Kowal D, Policar M, editors. Contraceptive Technology: 20th ed. New York, NY: Ardent Media; 2011.

5. World Health Organization. Medical Eligibility Criteria for Contraceptive Use, 5th ed. Geneva: World Health Organization; 2015.

6. NVivo qualitative data analysis software; QSR International Pty Ltd. Version 10, 2014

7. Patton MQ. Qualitative Research and Evaluation Methods, 3rd ed. Thousand Oaks: Sage; 2002.

8. Ulin PR, Robinson ET, Tolley EE. Qualitative methods in public health: a field guide for applied research, 1st ed. San Francisco, CA: Wiley; 2005.

9. Berger-Kulemann V, Einspeiler H, Hachemian N, Prayer D, et al. Magnetic field interactions of copper-containing intrauterine devices in 3.0 tesla magnetic resonance imaging: in vivo study. Korean J Radiol. 2013;14(3):416-422.

10. Richter L, Rotheram-Borus MJ, Van Heerden A, et al. Pregnant women living with HIV (WLH) supported at clinics by peer WLH: a cluster randomized controlled trial. AIDS Behav. 2014;18(4):706-715.

11. Gabitova G, Burke NJ. Improving healthcare empowerment through breast cancer patient navigation: a mixed methods evaluation in a safety-net setting. BMC Health Ser Res. 2014;14:407.

12. Güth I, Huang DJ, Bitzer J, Tirri BF, Moffat R. Contraception counseling for young breast cancer patients: A practical needs assessment and a survey among medical oncologists. Breast. 2016;30:217-221.
International Journal of Women's Health

\section{Publish your work in this journal}

The International Journal of Women's Health is an international, peerreviewed open-access journal publishing original research, reports, editorials, reviews and commentaries on all aspects of women's healthcare including gynecology, obstetrics, and breast cancer. The manuscript management system is completely online and includes

\section{Dovepress}

a very quick and fair peer-review system, which is all easy to use. Visit http://www.dovepress.com/testimonials.php to read real quotes from published authors. 